\title{
EVOLUTION OF LOW MASS BINARIES UNDER THE INFLUENCE OF GRAVITATIONAL RADIATION
}

\begin{abstract}
A.G. Massevitch, A.V. Tutukov, and L.R. Yungelson Astronomical Council, USSR Academy of Sciences
\end{abstract}

Evolutionary changes of masses and periods under the influence of gravitational radiation (GR) are computed for binaries with main-sequence or degenerate hydrogen-helium ( $\mathrm{H}$ ), helium (He) and carbon (C) secondaries. Tracks in the $\mathrm{P}-\mathrm{M}_{2}$ and $\mathrm{P}-\dot{\mathrm{M}}_{2}$ planes are determined. The orbital period of systems $P$ with a non-degenerate dwarf filling its Roche lobe decreases until the time-scale of GR $\left(\sim 10^{9}-10^{10}\right.$ yrs $)$ becomes shorter than the thermal time-scale $\tau_{\mathrm{KH}}$ of the mass losing component $\mathrm{M}_{2}$. When $\mathrm{M}_{2}$ becomes $\sim 0.1 \mathrm{M}_{\odot}$, $\underline{\mathrm{P}}$ begins to increase. This could account for the existence of the minimal $P \approx 1.3^{\mathrm{h}}$ and for the accumulation of observed cataclysmic binaries $(C B)$ below $P \leq 2^{h}$. The GR can be the driving force for evolution of cataclysmic binaries: TT Ari, OY Car, Z Cha, and WZ Sge as their $\underline{P}, M_{2}$ and $\dot{M}_{2}$ indicate. The rate of mass exchange driven by $G R$ is $\sim 10^{-9}-10^{-10} \mathrm{M}_{\odot} / \mathrm{yrs}$. It is enough to feed the $\mathrm{X}$-ray bursters and related objects.

In a binary with a degenerate secondary and initial $\mathrm{M}_{2} / \mathrm{M}_{1} \geq 0.6$ the secondary may transform into a disk around the primary during several orbital periods, liberating the energy of $\sim 10^{50}$ ergs. It appears that close binaries with both components degenerate may be the most powerful source of background GR near the Earth: $F \approx 10^{-5}-10^{-6}$ $\mathrm{erg} / \mathrm{cm}^{2} / \mathrm{sec}$.

The secondaries of $\mathrm{CB}$ have deep convective envelopes. Stellar winds are inherent in such stars, which may lead to a substantial angular momentum loss through magnetic braking. If the time-scale of the angular momentum loss is $10^{-9}-10^{-10}$ yrs, the resulting evolution of $\mathrm{CB}$ will be similar to that dominated by GR.

\section{DISCUSSION}

Van den Heuvel: Can you explain the very high $\mathrm{x}$-ray luminosities of the $\sim 20 \mathrm{X}$-ray sources in the galactic bulge, which have $L_{X}>10^{37} \mathrm{erg} / \mathrm{sec}$, requiring $10^{-9} \mathrm{M}_{\odot} / \mathrm{Yr}$ mass transfer? To my recollection, gravitational radiation-driven mass transfer from a red dwarf companion can never give

D. Sugimoto, D. Q. Lamb, and D. N. Schramm (eds.), Fundamental Problems in the Theory of Stellar Evolution, $185 \ldots 186$. Copyright $\odot 1981$ by the IAU. 
more than a few times $10^{-10} \mathrm{M}_{\odot} / \mathrm{Yr}$.

Tutukov: The mass exchange rate strongly depends on the assumed massradius relation. But up to now theory and observation can not provide us with reliable information about it. Our models show that a mass exchange rate in the range $10^{-9}-10^{-10} \mathrm{M}_{\odot} \mathrm{Yr}^{-1}$ is possible. To me, it seems possible that a black hole can sometimes be one component of such a system. It is possible also that a magnetic stellar wind might sometimes provide the mass exchange.

Van den Heuvel: I would like to point out that ostriker and źytkov, and Rappaport and Joss are independently doing similar calculations and are obtaining similar results, namely when the companion mass becomes $\lesssim 0.1 M_{\odot}$ the system expands again.

Tutukov: That is natural because of increasing interest in low mass binaries in the last few years.

Sato: What is the final state of a system evolving due to the emission of gravitational radiation? Is it the collapse or the destruction of the binary system?

Tutukov: Systems initially having main-sequence components and evolving due to gravitational radiation finish their evolution as binaries with component masses $\sim 0.03 \mathrm{M}_{\odot}+\sim 1 \mathrm{M}_{\odot}$ and a period $\sim 1.5$. Close binaries with degenerate somponents and having an initial mass ratio $q \geqslant 0.6$ transform into single stars surrounded by a heavy disk in a few orbital timescales. The same systems, but having $q \leqslant 0.6$, remain binaries with component masses $\sim 3 \times 10^{-3} \mathrm{M}_{\odot}+\sim \mathrm{M}_{\odot}$ (see Figure 1 in my review talk). 